\section{'Rapid access' chest pain clinics identify patients at high risk of cardiovascular events}

No-appointment 'rapid access' chest pain clinics are increasingly being used for the identification of first cases of angina, a primary manifestation of coronary heart disease. The success of these clinics in correctly differentiating between patients with angina-who require further cardiac assessment and treatment-and those with noncardiac chest pain has been investigated in a large-scale study performed in six UK centers.

Sekhri and co-workers in London, UK, analyzed data from 8,762 patients with new, undifferentiated chest pain referred from primary care to rapid access chest pain facilities. They found that patients diagnosed with angina in the rapid access centers ( $27 \%$ of referred patients) had a much higher risk of death from coronary heart disease or nonfatal acute coronary syndromes than patients diagnosed with noncardiac chest pain, and a higher risk than the general population. Among patients diagnosed with angina, the cumulative mortality resulting from coronary heart disease or acute coronary syndromes was estimated at $8.62 \%$ after 1 year, and $16.52 \%$ after 3 years, thereby strengthening evidence for a strong relationship between angina and future adverse coronary events.

Tarnishing these positive results, however, was the fact that nearly a third of the primary endpoints analyzed occurred in patients diagnosed with noncardiac chest pain; of these patients, those with diabetes, some Asians, and those with abnormalities on electrocardiography were at particularly high risk. The authors highlight the need for studies aimed at improving diagnostic precision, and for medics to initiate appropriate treatment for the prevention of future coronary events.

Original article Sekhri N et al. (2006) How effective are rapid access chest pain clinics? Prognosis of incident angina and non-cardiac chest pain in 8762 consecutive patients. Heart [doi: 10.1136/hrt.2006.090894]

\section{Fish oil does not seem to protect against ventricular arrhythmia in patients with ICDs}

Sudden cardiac death is a common manifestation of coronary heart disease. Omega-3 verylong-chain polyunsaturated fatty acids (omega-3
PUFAs), present in fish oil, are thought to reduce the risk of sudden cardiac death, possibly by lowering the risk of cardiac arrhythmia, but findings from studies are inconclusive. The Study on Omega-3 Fatty Acids and Ventricular Arrhythmia (SOFA) investigated whether omega-3 PUFAs affected the incidence of recurrent ventricular arrhythmia and all-cause mortality in 546 patients with implantable cardioverter-defibrillators (ICDs).

Patients were randomized to receive either $2 \mathrm{~g} /$ day of fish oil containing omega-3 PUFAs $(n=273)$, or placebo $(n=273)$, for approximately 12 months. The primary endpoint-death from any cause, or appropriate ICD intervention for spontaneous ventricular tachycardia or ventricular fibrillation-occurred in 81 patients (30\%) taking fish oil and in 90 patients (33\%) taking placebo. ICD intervention for tachyarrhythmia occurred in similar percentages of patients in the fish oil and placebo groups ( $27 \%$ vs $30 \%$, respectively). In patients who had previously had a myocardial infarction, there was a trend towards longer event-free survival in patients taking fish oil, but the difference between the groups was not significant.

The authors conclude that they did not find evidence of a strong protective effect of supplementation with omega-3 PUFAs against ventricular arrhythmias in patients with ICDs.

Original article Brouwer IA et al. (2006) Effect of fish oil on ventricular tachyarrhythmia and death in patients with implantable cardioverter defibrillators: the Study on Omega-3 Fatty Acids and Ventricular Arrhythmia (SOFA) randomized trial. JAMA 295: 2613-2619

\section{Tissue-specific gene mutations might predispose individuals to idiopathic AF}

Most molecular pathology studies of atrial fibrillation (AF) have focused on rare, familial forms of the disorder. While these analyses are likely to provide insights into the molecular pathways involved in AF, the genetic abnormalities identified might not represent the pathogenesis in more-common, nonfamilial forms of AF. In their study, Gollob et al. investigated whether idiopathic AF has a genetic basis. Since tissue-specific and somatic mutations are increasingly being identified as the cause of sporadic cases of nonmalignant disease, they also hypothesized that mutations 